\title{
Thermal Transport between Graphene Sheets and SiC Substrate by Molecular-Dynamical Calculation
}

\author{
Zan Wang, ${ }^{1}$ Kedong Bi, ${ }^{2}$ Huawei Guan, ${ }^{1}$ and Jiong Wang ${ }^{1}$ \\ ${ }^{1}$ Henan University of Technology, Zhengzhou 450007, China \\ ${ }^{2}$ Southeast University, Nanjing 211102, China \\ Correspondence should be addressed to Zan Wang; truewz@163.com
}

Received 4 January 2014; Accepted 7 February 2014; Published 23 April 2014

Academic Editor: Necmettin Maraşlı

Copyright (c) 2014 Zan Wang et al. This is an open access article distributed under the Creative Commons Attribution License, which permits unrestricted use, distribution, and reproduction in any medium, provided the original work is properly cited.

\begin{abstract}
Using nonequilibrium molecular dynamics, we investigate the mechanisms of thermal transport across SiC/graphene sheets. In simulations, 3C-, $4 \mathrm{H}-$, and $6 \mathrm{H}-\mathrm{SiC}$ are considered separately. Interfacial thermal resistances between Bernal stacking graphene sheets and $\mathrm{SiC}$ (Si- or C-terminated) are calculated at the ranges of $100 \mathrm{~K} \sim 700 \mathrm{~K}$. The results indicate, whether Si-terminated or $\mathrm{C}$-terminated interface, the interfacial thermal resistances of $4 \mathrm{H}$ - and $6 \mathrm{H}$-SiC have similar trends over temperatures. Si-terminated interfacial thermal resistances of $3 \mathrm{C}$ - $\mathrm{SiC}$ are higher than those of $4 \mathrm{H}$ - and $6 \mathrm{H}-\mathrm{SiC}$ in a wide temperature range from $100 \mathrm{~K}$ to $600 \mathrm{~K}$. But, for C-rich interface, this range is reduced from $350 \mathrm{~K}$ to $500 \mathrm{~K}$.
\end{abstract}

\section{Introduction}

For the atomically thin structure of graphene, it has attracted much attention due to potential applications to thermoelectric and photoelectric devices. Over the past decade, researchers mainly focus on the fabrication of graphene and its physical characteristics. The most common used methods of graphene fabrication are epitaxial growth on silicon carbide ( $\mathrm{SiC}$ ) and chemical vapor deposition (CVD). As to epitaxial growth method, Si atoms sublimate from a single-crystal $\mathrm{SiC}$ substrate and create large area graphene sheets. CVD method employs carbon source gas to react with the specific substrate chemically, so as to synthesize graphene sheets. In recent years, the techniques of graphene production have been improved dramatically. Tzalenchuk et al. [1] synthesized $50 \mathrm{um}^{2}$ graphene sheets by epitaxial growth method. Soon after, Bae et al. [2] made graphene films with $762 \mathrm{~mm}$ diagonal length onto $\mathrm{Cu}$ substrate. In 2011, Zhang et al. [3] first realized the low temperature growth of graphene onto several substrates such as $\mathrm{SiC}$, $\mathrm{SiO}_{2}$, and $\mathrm{Al}_{2} \mathrm{O}_{3}$ by plasma enhancement chemical vapor deposition (PECVD). Through solid-state molecular beam epitaxy (SSMBE), Tang et al. [4] made graphene films grown on $\mathrm{Si}(111)$ substrate and analyzed the effects of $\mathrm{SiC}$ buffer layer on stabilizing the surface of Si substrate. Janssen et al. [5-7] investigated the Hall resistance in epitaxial graphene grown on Si-terminated $\mathrm{SiC}$ and other graphene material systems. At present, although numerous studies have demonstrated the high thermal conductivities of graphene and graphene sheets along tangent direction, little work has been done to address the characteristics of graphene sheets along normal direction, let alone $\mathrm{SiC} /$ graphene composite films.

\section{Theory}

As to theoretical study, researchers usually adopt analytical or numerical approaches to perform nanoscale heat transport simulations, involving molecular dynamics (MD) method, Monte Carlo (MC) method, Boltzmann transport equation, and Green's function. Compared with other methods, MD is much closer to physical reality, which defines the simulation region with atoms, supposed to the arrangement of chemical structures. Based on Newton's law, MD sets cut-off radius to build atom groups, in which atoms interact with each other by elastic forces. At the beginning of MD simulation, motion parameters of each atom should be initialized according to the imposed temperatures. After enough circulations, system reaches to an equilibrium and the temperature can be obtained by Boltzman equipartition theorem (BET). 


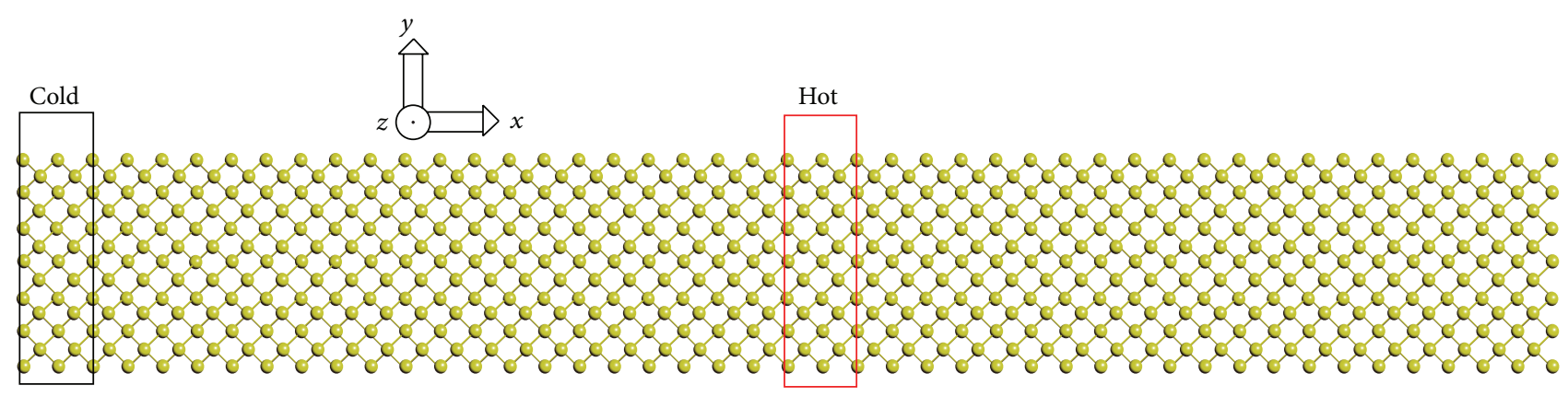

FIGURE 1: Ball and stick model of 3C-SiC film in this work.

According to the different calculation principles, MD can be divided into equilibrium MD (EMD) and nonequilibrium MD (NEMD) methods. As to EMD, thermal conductivity may be obtained by Green-Kubo linear response under equilibrium state [8]. For NEMD, through modulating the temperature of hot bath and cold bath, thermal flux is created and temperature gradient emerges in the system, and then thermal conductivity can be evaluated by Fourier's theorem $[9,10]$. NEMD method is analogous to the experimental measurements of heat conduction and is preferred over the EMD-based approach to compute thermal conductivity of inhomogeneous material systems $[11,12]$. Recently, low-sized nanoscale materials, such as core-shell nanowires, superlattice, graphene, and nanotube [13-22], were investigated by NEMD method. In this paper, we use a NEMD-based approach to study the thermal performance of $\mathrm{SiC}$ /graphene composite films.

The key points of NEMD method are the creation of thermal flux and the interaction potential. In order to create thermal flux, we may keep the temperature gap between the hot part and the cold part, which drives thermal flux. Another way is to swap the coldest atoms in the hot part with the hottest ones in cold part. From the computation viewpoint, the latter is more effective, because the number of atoms involved in the calculation is less than the former. Therefore, we utilize the former way to drive thermal flux in NEMD simulations.

In semiconductors, heat transfer mainly depends on the transmission of elastic vibration by interatomic force, which is the derivative of the interaction potential. Hence, whether the right interaction potential is utilized determines the correctness of the results. In this work, Tersoff [23, 24], Airebo [2], and Lennard-Jones (LJ) functions are used together to construct interaction potential energy model. Tersoff potential as three-body interaction function is adopted to calculate the interatomic force of $\mathrm{Si}-\mathrm{C}$ covalent bond in $\mathrm{SiC}$ material. Airebo and Lennard-Jones functions belong to twobody potential interaction. For a single sheet of graphene, the $\mathrm{C}-\mathrm{C}$ short-range force can be solved by the former. The latter is used to evaluate $\mathrm{C}-\mathrm{C}$ long-range force between the graphene sheets.

A NEMD ball and stick 3C-SiC film model in our study is depicted in Figure 1. Along $x, y$, and $z$ direction, this model has three periodic boundary conditions. Hot zone is set in the middle and cold zone is set to the left part. Heat flux flows from the hot zone to the left side and right side. After equilibrium, temperature gradient may be created in this model.

In Figure 2, the results of 3C-SiC, graphene, and Si films by NEMD method are compared to confirm its validity. The cross section of this system is $6 \times 8$ unit cells, with a length of 140 unit cells at $100 \mathrm{~K}$. We set the time step as $0.766 \mathrm{fs}$ and the average temperature of this system equals $100 \mathrm{~K}$. The processes of this simulation can be divided into two stages. The first stage is to relax atoms, and the next one is to establish stable temperature gradient. As shown in Figure 2, for 3C-SiC, graphene, and Si films, the fitting curve of temperature distribution shows a good linear dependence of cell serial number. Because of the interface scattering, there exist temperature jumps prominently near the hot bath and cold bath [11]. The right inset figure in Figure 2 shows the heat flux, which equals the ratio of the total transferred kinetic energy to time and the cross-sectional area.

Exchanging kinetic energy of atoms in hot bath and cold bath, system reaches to an equilibrium state and thermal conductivity can be obtained from the following equation:

$$
\lambda=q \cdot \frac{1}{\nabla T}=-\frac{\sum_{\text {transfrer }}(1 / 2)\left(M v_{h}^{2}-m v_{c}^{2}\right)}{2 t L_{y} L_{z}} \cdot \frac{1}{\langle\partial T / \partial x\rangle},
$$

where $q$ denotes heat flux, $\nabla T$ is the gradient of temperature profile, $L_{y} L_{z}$ represents the area of cross section normal to thermal flux, $M(m)$ is the mass of atom in the cold bath (hot bath), $t$ is the simulation time, and $v_{h}\left(v_{c}\right)$ indicates the velocity of the coldest (hotest) atoms in hot bath (cold bath). We also calculate the in-plane thermal conductivities of monolayer graphene on $\mathrm{SiO}_{2}$ substrate, as shown in Figure 3. In this NEMD model, Tersoff, Airebo, and LJ potential functions referring to Zhao's work [25] are employed to calculate the chemical bonding interactions of $\mathrm{C}-\mathrm{C}, \mathrm{Si}-\mathrm{Si}$, $\mathrm{Si}-\mathrm{O}$, and O-O. In simulations, NEMD results are about $50 \%$ lower than the experimental values [26], which is perhaps due to quantum errors between realistic temperatures and molecular dynamics temperatures. Thus, the proximity between the experimental values and the NEMD results confirms the validity of the physical model we built. 


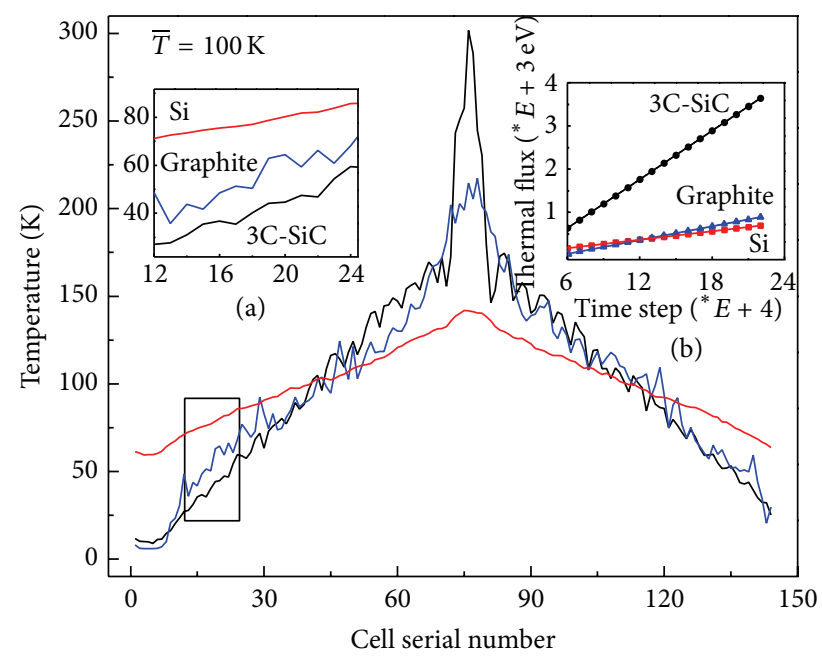

Figure 2: Temperature distributions of 3C-SiC, graphene sheets, and Si across cell serial number. (a) The enlarged figure. (b) The thermal flux.

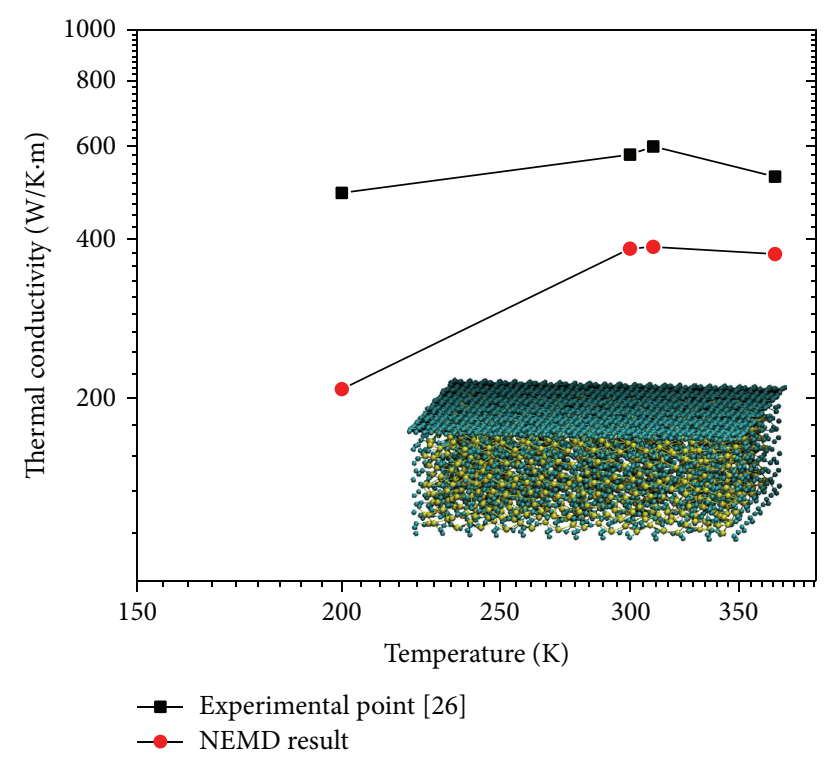

FIGURE 3: In-plane thermal conductivities of monolayer graphene on $\mathrm{SiO}_{2}$ substrate.

Interfacial thermal resistance $R$ can be calculated from

$$
R=\frac{\Delta T}{q}
$$

where $\Delta T$ is the temperature drop at the interface of graphene/SiC. Figure 4 shows the schematic diagram of a graphene/SiC composite film and the lattice unit cell structures of $3 \mathrm{C}-, 4 \mathrm{H}-$, and $6 \mathrm{H}-\mathrm{SiC}$ polytypes. In simulations, we utilized $\mathrm{AB}$ (Bernal) stacking graphene sheets in place of $\mathrm{AA}$, $\mathrm{ABC}$ stacking, because above $80 \%$ monocrystalline graphite belongs to $\mathrm{AB}$ stacking in reality $[17,27]$.

The lattice parameters used in our work are listed in Table 1. $a, b$, and $c$ are the lengths of the three axial-vectors.
TABLE 1: Lattice parameters of SiC polytypes and graphene [28].

\begin{tabular}{lcccc}
\hline & $3 \mathrm{C}-\mathrm{SiC}$ & $4 \mathrm{H}-\mathrm{SiC}$ & $6 \mathrm{H}-\mathrm{SiC}$ & Graphene \\
\hline$a=b(\AA)$ & 4.348 & 3.073 & 3.0813 & 2.552 \\
$C(\AA)$ & 4.348 & 10.0848 & 15.1198 & 3.35 \\
\hline
\end{tabular}

In Figure 5, we build a model of 4H-SiC/graphene sheets and calculate its temperature distribution, at the average temperature of $300 \mathrm{~K}$. Hot bath locates at the middle of graphene sheets and cold bath is set at the right end of $4 \mathrm{H}$ $\mathrm{SiC}$ film. The enlarged graph in Figure 5 shows a temperature drop at the interface due to the interfacial thermal resistance while on both sides of the interface, the fitting curve of temperature distribution is still linear. It is noteworthy that the profiles of temperature are not symmetrical and the values of $\Delta T$ on the two sides of the hot bath are different, which is because the interfacial morphologies are not identical strictly.

The lattice mismatch of $\mathrm{SiC} /$ graphene system can strongly affect the special thermal properties and electronic characteristics. Correspondingly, it is necessary to investigate the influences of different interfacial morphologies on thermal transports. As shown in Figure 6, we investigate the averaged interfacial thermal resistances between Si-terminated $\mathrm{SiC}$ polytypes and graphene sheets. Referring to the work of Hass and so forth, we set the distance between the first graphene layer and $\mathrm{Si}$ layer of $\mathrm{SiC}$ as $2 \sim 2.5 \AA$. From Figure $6,4 \mathrm{H}$ - and $6 \mathrm{H}-\mathrm{SiC}$ have similar convex increasing trends of interfacial thermal resistances. The interfacial thermal resistance of 3C-SiC initially increases with the increasing temperatures from $100 \mathrm{~K}$ to $300 \mathrm{~K}$ and then shows a downward bending above $300 \mathrm{~K}$. The results indicate $3 \mathrm{C}-\mathrm{SiC}$ has larger interfacial thermal resistances than those of $4 \mathrm{H}-$ and $6 \mathrm{H}-\mathrm{SiC}$ below $600 \mathrm{~K}$ and has an obvious peak value about $2.5 \times 10^{-9} \mathrm{Km}^{2} / \mathrm{W}$ at $300 \mathrm{~K}$. Over $400 \mathrm{~K}$, the values of $6 \mathrm{H}-\mathrm{SiC}$ similarly conserve to a constant about $1.75 \times 10^{-9} \mathrm{Km}^{2} / \mathrm{W}$. Different from $3 \mathrm{C}-\mathrm{SiC}$ and $6 \mathrm{H}-\mathrm{SiC}, 4 \mathrm{H}-\mathrm{SiC}$ keeps linear increasing trend from $100 \mathrm{~K}$ to $700 \mathrm{~K}$.

In the CVD graphene growing method, we can also use C-rich surface to bond with $\mathrm{C}$ atoms. The interface of $\mathrm{SiC} /$ graphene becomes different from Si-rich interface as depicted in Figure 7. Compared with Figure 6, the maximum values of the three $\mathrm{SiC}$ polytypes are larger than those in Figure 7. The curve of 3C-SiC complies with approximately Gauss distribution and the peak value locates at about $400 \mathrm{~K}$. The trend of $6 \mathrm{H}-\mathrm{SiC}$ is a little changed; however the interfacial thermal resistances of $4 \mathrm{H}-\mathrm{SiC}$ may not keep linear and show convex profile as $6 \mathrm{H}-\mathrm{SiC}$. We suggest the bond energy of $\mathrm{C}-$ $\mathrm{C}$ is stronger than that of $\mathrm{Si}-\mathrm{C}$ bond; that is, $\mathrm{C}-\mathrm{C}$ bond has larger potential energy. Therefore, $\mathrm{C}$-rich surface has bigger interfacial thermal resistances than Si-rich surface.

\section{Conclusion}

By chemical method, monolayer graphene and graphene sheets can be synthesized on $\mathrm{SiC}$ substrates. Many researchers have studied electrical and thermal properties of graphene; however, few works involve the thermal transport of $\mathrm{SiC} /$ graphene sheets. In this work, we apply a dedicated 


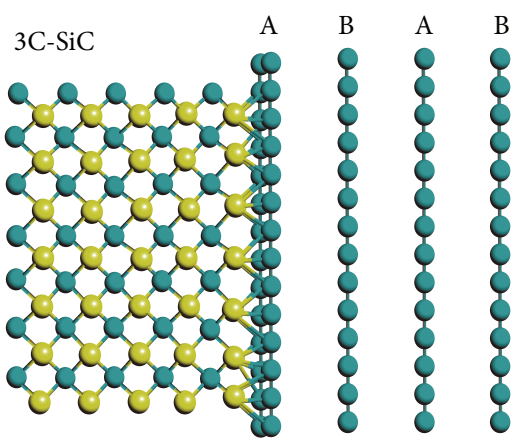

(a)
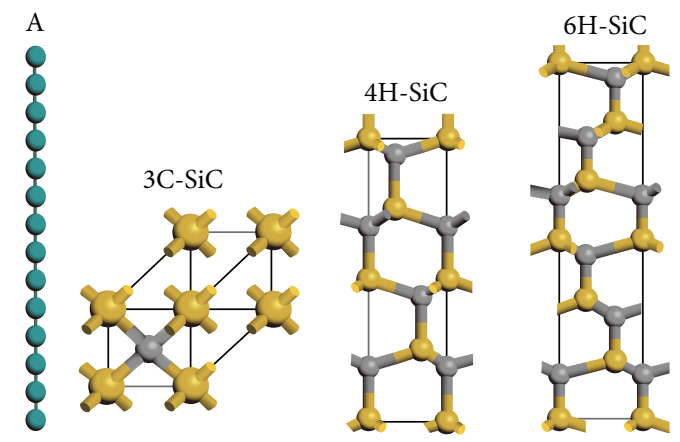

(b)

FIGURE 4: Schematic diagram of graphene sheets/SiC.

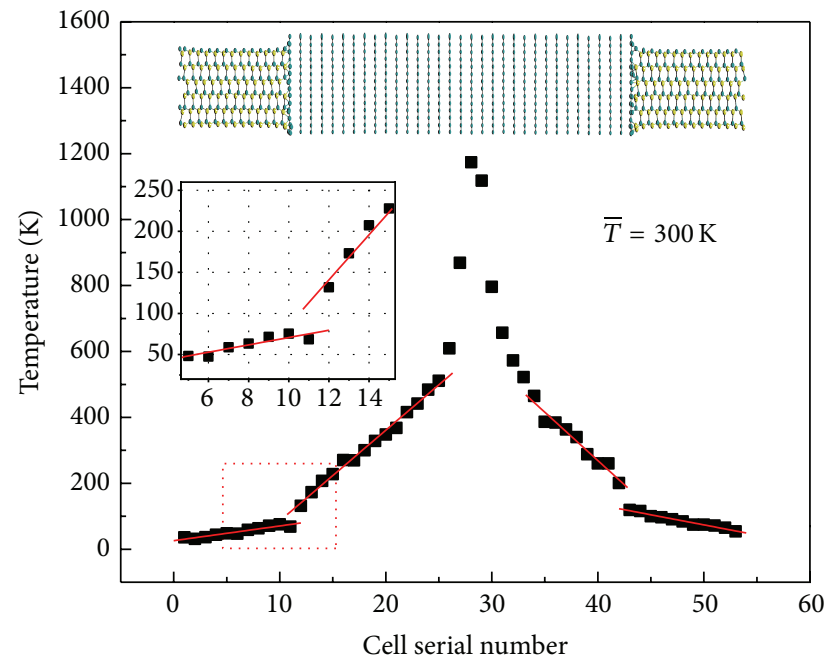

Figure 5: Temperature distribution of $4 \mathrm{H}-\mathrm{SiC} /$ graphene sheets across the length.

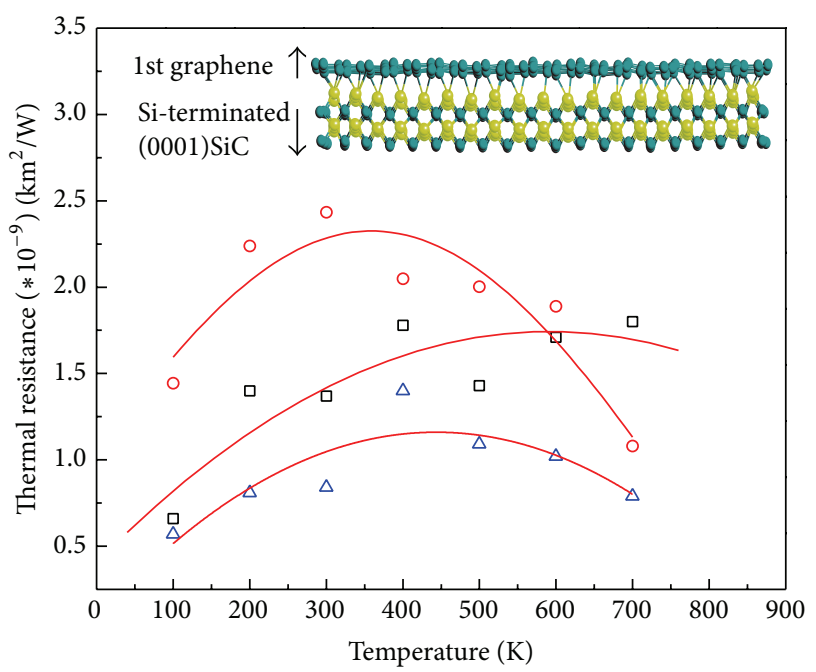
- $3 \mathrm{C}-\mathrm{SiC}$
$6 \mathrm{H}-\mathrm{SiC}$
$\triangle 4 \mathrm{H}-\mathrm{SiC}$

Figure 6: Interfacial thermal resistances of Si-teminated SiC films.

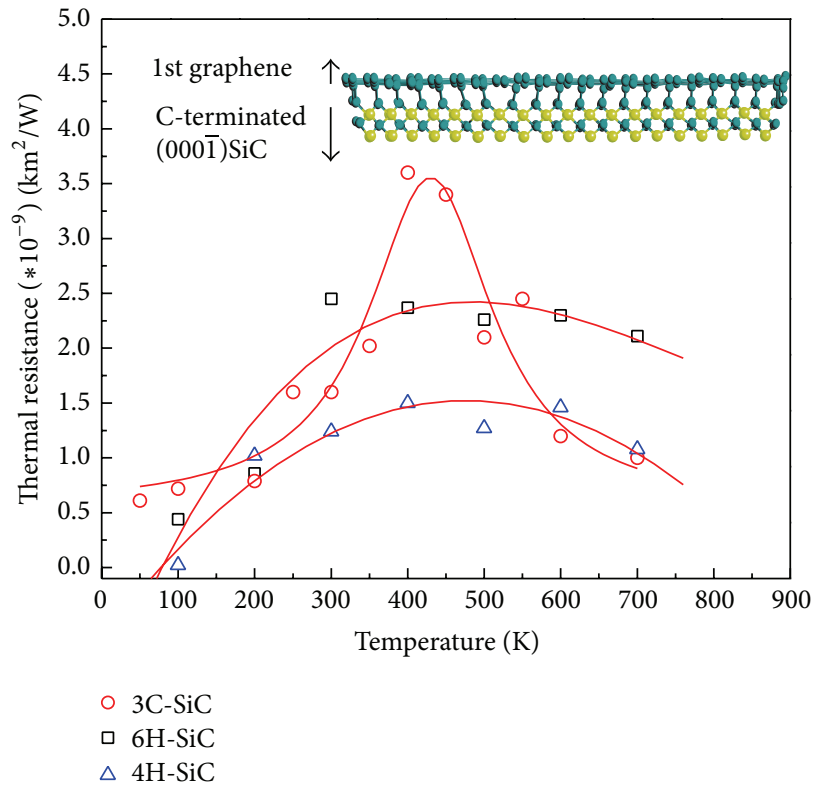

FIGURE 7: Interfacial thermal resistances of C-terminated 3C-, 4H, $6 \mathrm{H}-\mathrm{SiC} /$ graphene sheets across temperatures.

NEMD model to investigate interfacial thermal resistances of SiC/graphene sheets. Three polytypes of $\mathrm{SiC}(3 \mathrm{C}-, 4 \mathrm{H}-$, and $6 \mathrm{H}-\mathrm{SiC}$ ) are set as substrates to bond with graphene sheets in simulation. Si-rich and C-rich interfaces between $\mathrm{SiC/graphene} \mathrm{are} \mathrm{also} \mathrm{analyzed} \mathrm{at} \mathrm{the} \mathrm{temperatures} \mathrm{from}$ $100 \mathrm{~K}$ to $700 \mathrm{~K}$. From the results of Si-rich interface, the interfacial thermal resistance of $3 \mathrm{C}-\mathrm{SiC}$ is larger than those of $4 \mathrm{H}$ - and $6 \mathrm{H}-\mathrm{SiC}$ below $600 \mathrm{~K}$. From $100 \mathrm{~K}$ to $700 \mathrm{~K}$, the curve of $6 \mathrm{H}-\mathrm{SiC}$ is always greater than that of $4 \mathrm{H}-\mathrm{SiC}$. By fitting the calculated datum, the peak interfacial thermal resistances of $3 \mathrm{C}-, 4 \mathrm{H}-$, and $6 \mathrm{H}-\mathrm{SiC}$ are $2.3 \times 10^{-9} \mathrm{Km}^{2} / \mathrm{W}$ (at $354 \mathrm{~K}$ ), $1.74 \times 10^{-9} \mathrm{Km}^{2} / \mathrm{W}$ (at $571 \mathrm{~K}$ ), and $1.16 \times 10^{-9} \mathrm{Km}^{2} / \mathrm{W}$ (at $457 \mathrm{~K})$. As to C-rich interface in Figure 7, the trends of $4 \mathrm{H}$ and $6 \mathrm{H}-\mathrm{SiC}$ are not changed greatly, but the curve of $3 \mathrm{C}-\mathrm{SiC}$ approximates to Gauss distribution and its maximum reaches to $2.95 \times 10^{-9} \mathrm{Km}^{2} / \mathrm{W}$ (at $480 \mathrm{~K}$ ). Especially, the values of $3 \mathrm{C}-\mathrm{SiC}$ become lower than those of $3 \mathrm{C}-\mathrm{SiC}$ and $4 \mathrm{H}-\mathrm{SiC}$ over $600 \mathrm{~K}$. 
However, it should be acknowledged that surface reconstructions of $\mathrm{SiC}$ such as $(3 \times 3),(\sqrt{3} \times \sqrt{3}) R 30^{\circ}$, and $(6 \sqrt{3} \times$ $6 \sqrt{3}) R 30^{\circ}$ are not considered in this work, which needs to be further investigated in the near future. We expect to give a theory reference to the applications of SiC/graphene films in the field of micro/nanothermoelectric devices.

\section{Conflict of Interests}

The authors declare that there is no conflict of interests regarding the publication of this paper.

\section{Acknowledgments}

Zan Wang would like to acknowledge the financial support from the National Natural Science Foundation of China (51106043, 51205061, 51373048), and this work is also supported by the basic research project of Henan Provincial Department of Education Science and Technology (13A470176) and Plan For Scientific Innovation Talent of Henan University of Technology.

\section{References}

[1] A. Tzalenchuk, S. Lara-Avila, A. Kalaboukhov et al., "Towards a quantum resistance standard based on epitaxial graphene," Nature Nanotechnology, vol. 5, no. 3, pp. 186-189, 2010.

[2] S. Bae, H. Kim, Y. Lee et al., "Roll-to-roll production of 30-inch graphene films for transparent electrodes," Nature Nanotechnology, vol. 5, no. 8, pp. 574-578, 2010.

[3] L. C. Zhang, Z. W. Shi, Y. Wang, R. Yang, D. Shi, and G. Zhang, "Catalyst-free growth of nanographene films on various substrates," Nano Research, vol. 4, no. 3, pp. 315-321, 2011.

[4] J. Tang, C. Y. Kang, L. M. Li, W. S. Yan, S. Q. Wei, and P. S. Xu, "Graphene films grown on Si substrate via direct deposition of solid-state carbon atoms," Physica E: Low-Dimensional Systems and Nanostructures, vol. 43, no. 8, pp. 1415-1418, 2011.

[5] T. J. B. M. Janssen, A. Tzalenchuk, R. Yakimova et al., "Anomalously strong pinning of the filling factor $\nu=2$ in epitaxial graphene," Physical Review B: Condensed Matter and Materials Physics, vol. 83, no. 23, Article ID 233402, 2011.

[6] T. J. B. M. Janssen, N. E. Fletcher, R. Goebel et al., "Graphene, universality of the quantum Hall effect and redefinition of the SI system," New Journal of Physics, vol. 13, Article ID 093026, 2011.

[7] T. J. B. M. Janssen, A. Tzalenchuk, S. L. Avila et al., "Quantum resistance metrology using graphene," Reports on Progress in Physics, vol. 76, no. 10, Article ID 104501, 2013.

[8] M. P. Allen and D. J. Tildesley, Computer Simulation of Liquids, Clarendon Press, Oxford, UK, 1987.

[9] F. C. Chou, J. R. Lukes, X. G. Liang et al., "Molecular dynamic in microscale thermophysical engineering," Annual Review of Heat Transfer, vol. 10, pp. 141-176, 1999.

[10] F. Müller-Plathe, "A simple nonequilibrium molecular dynamics method for calculating the thermal conductivity," Journal of Chemical Physics, vol. 106, no. 14, pp. 6082-6085, 1997.

[11] P. K. Schelling, S. R. Phillpot, and P. Keblinski, "Comparison of atomic-level sim ulation methods for computing thermal conductivity," Phyical Review B, vol. 65, no. 14, Article ID 144306, 2002.
[12] M. H. Khadem and A. P. Wemhoff, "Comparison of GreenKubo and NEMD heat flux formulations for thermal conductivity prediction using the Tersoff potential," Computational Materials Science, vol. 69, pp. 428-434, 2013.

[13] S. Ju, X. Liang, and X. Xu, "Out-of-plane thermal conductivity of polycrystalline silicon nanofilm by molecular dynamics simulation," Journal of Applied Physics, vol. 110, no. 5, Article ID 054318, 2011.

[14] X. P. Huang, X. L. Huai, S. Q. Liang et al., “Thermal transport in Si/Ge nanocomposites," Journal of Physics D: Applied Physics, vol. 42, no. 9, Article ID 095416, 2009.

[15] S.-C. Wang, X.-G. Liang, X.-H. Xu, and T. Ohara, “Thermal conductivity of silicon nanowire by nonequilibrium molecular dynamics simulations," Journal of Applied Physics, vol. 105, no. 1, Article ID 014316, 2009.

[16] X. M. Yang, A. C. To, and R. Tian, "Anomalous heat conduction behavior in thin finite-size silicon nanowires," Nanotechnology, vol. 21, no. 15, Article ID 155704, 2010.

[17] M. Hu, K. P. Giapis, J. V. Goicochea, X. L. Zhang, and D. Poulikakos, "Significant reduction of thermal conductivity in Si/Ge core-shell nanowires," Nano Letters, vol. 11, no. 2, pp. 618623, 2011.

[18] G. Wu and B. W. Li, "Thermal rectification in carbon nanotube intramolecular junctions: molecular dynamics calculations," Physical Review B, vol. 76, no. 8, Article ID 085424, 2007.

[19] N. Yang, G. Zhang, and B. W. Li, "Carbon nanocone: a promising thermal rectifier," Applied Physics Letters, vol. 93, no. 24, Article ID 243111, 2008.

[20] W. R. Zhong, W. H. Huang, X. R. Deng, and B. Q. Ai, “Thermal rectification in thickness asy mmetric graphene nanoribbons," Applied Physics Letters, vol. 99, no. 19, Article ID 193104, 2011.

[21] T. F. Luo and J. R. Lloyd, "Enhancement of thermal energy transport across graphene/graphite and polymer interfaces: a molecular dynamics study," Advanced Functional Materials, vol. 22, no. 12, pp. 2495-2502, 2012.

[22] V. Samvedi and V. Tomar, "Role of heat flow direction, monolayer film thickness, and periodicity in controlling thermal conductivity of a Si-Ge superlattice system," Journal of Applied Physics, vol. 105, no. 1, Article ID 013541, 2009.

[23] J. Tersoff, "Modeling solid-state chemistry: interatomic potentials for multicomponent systems," Physical Review B, vol. 39, no. 8, Article ID 5566, 1989.

[24] D. W. Brenner, "Empirical potential for hydrocarbons for use in simulating the chemical vapor deposition of diamond films," Physical Review B, vol. 42, no. 15, Article ID 9458, 1990.

[25] S. J. Zhao, J. M. Xue, Y. G. Wang et al., "Effect of $\mathrm{SiO}_{2}$ substrate on the irradiation-assisted manipulation of supported graphene: a molecular dynamics study," Nanotechnology, vol. 23, no. 28, Article ID 285703, 2012.

[26] M. T. Pettes, X. S. Li, Z. Yao et al., "Two-dimensional phonon transport in supported graphene," Science, vol. 328, no. 5975, pp. 213-216, 2010.

[27] J. Hass, W. A. de Heer, and E. H. Conrad, "The growth and morphology of epitaxial multilayer graphene," Journal of Physics Condensed Matter, vol. 20, no. 32, Article ID 323202, 2008.

[28] A. B. J. Kräußlich, L. Dressler, P. Kuschnerus et al., "Highprecision determination of atomic positions in crystals: the case of $6 \mathrm{H}-$ and $4 \mathrm{H}-\mathrm{SiC}$," Physical Review B, vol. 57, no. 5, pp. 26472650, 1998. 

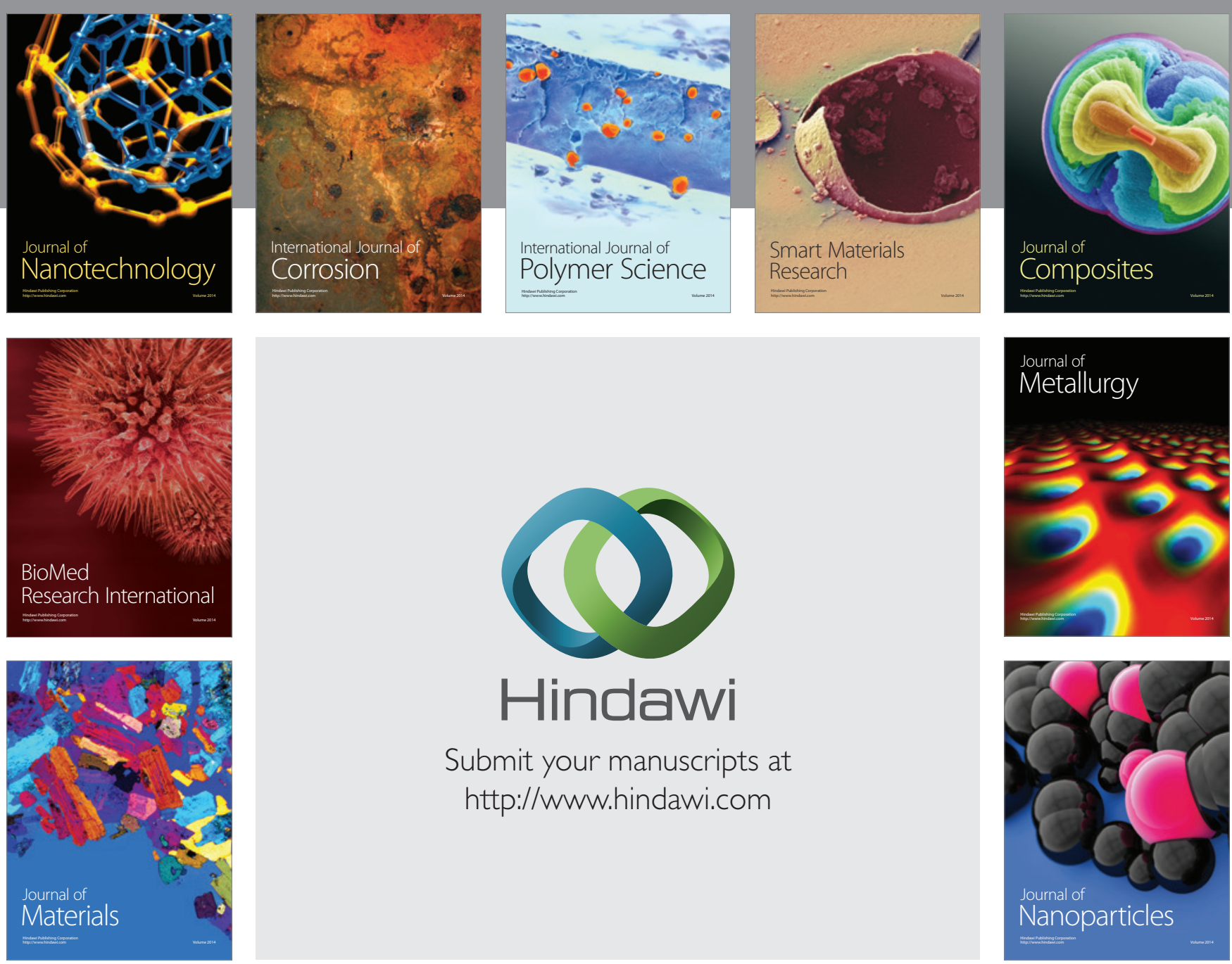

Submit your manuscripts at http://www.hindawi.com
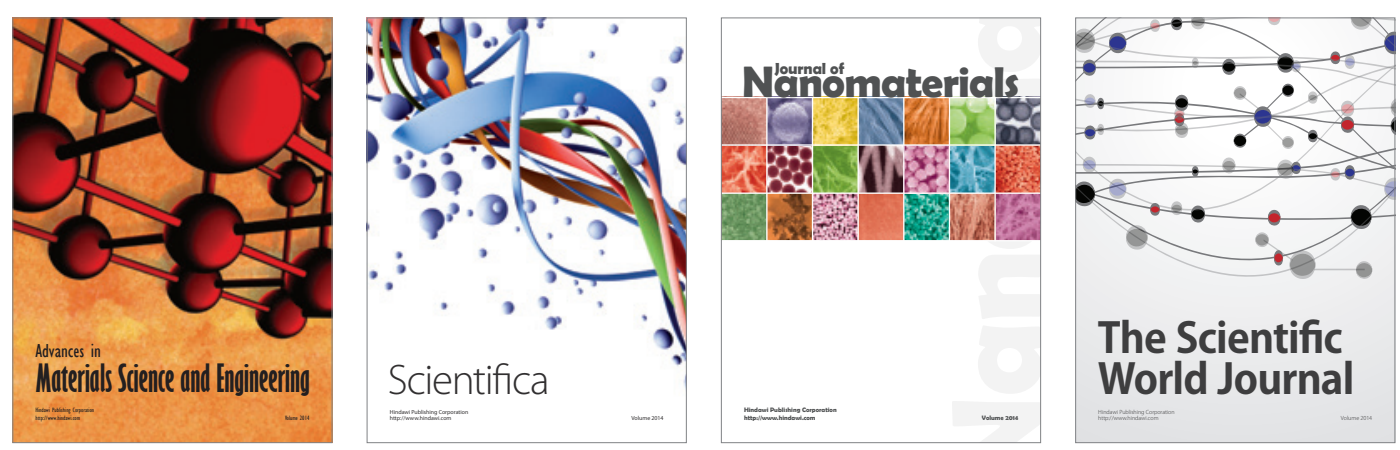

\section{The Scientific World Journal}
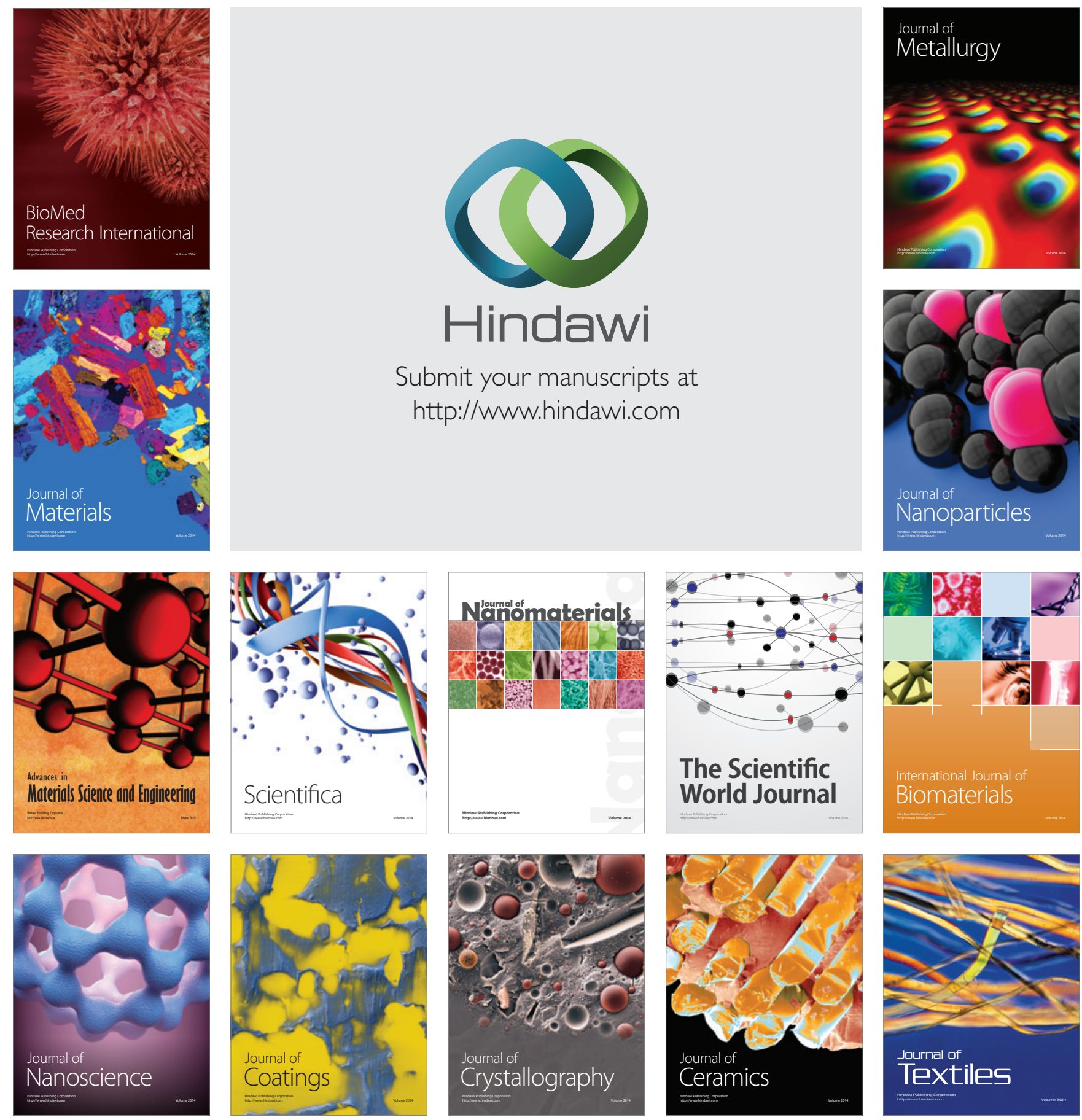